\title{
Una Metodología Automatizada para la Evaluación de Usabilidad de Interfaces de Supervisión Industrial
}

\author{
Luini L. Hurtado(1), Omar D. Castrillón ${ }^{(2)}$ y Gerard Olivar ${ }^{(2)}$ \\ (1) Universidad Distrital Francisco José de Caldas, Carrera 7 No. 40B - 53, Santa Fe de Bogotá-Colombia \\ (e-mail: Ilhurtadoc@udistrital.edu.co) \\ (2) Universidad Nacional de Colombia, Sede Manizales, Carrera 27 No. 64 - 60, Manizales-Colombia \\ (e-mail:odcastrillong@unal.edu.co, golivart@unal.edu.co)
}

Recibido Ene. 23, 2013; Aceptado Mar. 11, 2013; Versión final recibida Abr. 29, 2013

\section{Resumen}

Se presenta el desarrollo, aplicación y validación de una metodología para la evaluación de la usabilidad de interfaces de sistemas de supervisión industrial. Se exponen los aspectos fundamentales de evaluación de usabilidad de sistemas basados en software y se explica la metodología. La metodología fue aplicada en un laboratorio a varias interfaces de supervisión de un proceso de dosificación de líquidos. El sistema es utilizado para determinar la valoración de la interfaz de supervisión de la interacción entre el operador y el proceso. Las valoraciones sirvieron para clasificar la usabilidad de las interfaces. Para comprobar la validez de la metodología, se realizó una prueba de hipótesis frente a otras dos metodologías tradicionales: inspección por el experto e indagación a los usuarios. Se determinó que la metodología propuesta es equivalente a los otros dos métodos, pero tiene la ventaja de ser completamente automatizada.

Palabras clave: usabilidad, sistemas de supervisión, interfaz humano-máquina, sistemas neuro-difuso

\section{An Automated Methodology for Usability Evaluation of Interfaces of Industrial Supervision}

\begin{abstract}
The development, application and validation of a methodology for usability evaluation of interfaces of industrial supervision systems are presented. The fundamental aspects of usability evaluation software based systems are described and the methodology is presented. The methodology was applied in a laboratory to monitor various interfaces of a liquid dosing process. The system is used to determine the value of the supervision interface for the interaction between the operator and the process. The ratings were useful for classifying interface usability. To check the validity of the methodology, a hypothesis test was done and results compared to two traditional methods: expert inspection and inquiry to users. It was determined that the proposed methodology is equivalent to the other two methods, but it has the advantage of being completely automated.
\end{abstract}

Keywords: usability, supervision systems, human-machine interface, neuro-fuzzy systems 


\section{INTRODUCCIÓN}

Existen diversas perspectivas teóricas y empíricas que encierra el término usabilidad para sistemas interactivos. Para ello, en primer lugar, se referencia desde lo expuesto por el estándar Ergonomic requirements for office work with visual display terminals (ISO 9241, 1998), que describe los requisitos ergonómicos para trabajo de oficina con terminales de despliegue visual y explica algunos de los principios básicos subyacentes. El estándar Guidance on Usability (ISO 9241-11, 1998) define aquellos factores que tienen un efecto en la usabilidad de productos software.

Según el estándar Software Product Evaluation - Quality Characteristics and Guidelines for the Use (ISO/IEC 25000:2005), la usabilidad es analizada en términos de su comprensibilidad, aprendizaje, operabilidad, atractividad y complacencia. La usabilidad es "la extensión para la que un producto puede ser usado por usuarios específicos, para lograr metas específicas con efectividad, eficacia y satisfacción en un contexto de uso específico" (ISO/DIS 9241-11). Pero existe otra conceptualización y teoría al respecto de la ISO, la cual presenta diversas perspectivas y planteamientos complementarios. En el estándar (ISO/IEC 25000:2005), la usabilidad es un atributo de la calidad del software, siendo la calidad del software definida por el estándar como un conjunto de atributos que se manifiestan por el esfuerzo necesario para el uso y por la valoración individual de tal uso por un conjunto de usuarios involucrados. Para el caso de productos de software, se plantean teorías que centran los modelos en personas (Norman, 1986; Wickens y Hollands, 2000; Anderson, 2000) y teorías de evaluación de usabilidad de las Interfaces (Rodeiro, 2001; Lorés et. al., 2001; Dix et. al., 2004).

Por otra parte, se analiza la evaluación, la cual consiste en probar algo, tanto para saber si funciona correctamente como no, si cumple con las expectativas o no, o simplemente para conocer cómo funciona una determinada herramienta o utensilio, la evaluación de la usabilidad es una actividad que comprende un conjunto de metodologías y técnicas que analizan la usabilidad de un sistema en diferentes etapas del ciclo de vida del software (Granollers et al., 2005). De la misma forma, se debe comprender que es necesario complementar la evaluación desde la perspectiva del usuario, utilizando métodos de indagación y desde la perspectiva del experto, utilizando métodos de inspección. Un excelente trabajo de revisión sobre varios aportes al concepto de usabilidad y su evaluación se recoge en (Ankita y Sanjay, 2012), allí, en una de las referencias, se plantean diez factores de usabilidad y veintiséis criterios de evaluación (Seffah et al., 2006), que posteriormente son agrupados y plasmados en una taxonomía (Rios et al., 2010).

Recientemente, se ha hecho evidente la preocupación por la evaluación de la usabilidad y calidad del software de uso industrial. Se han propuesto enfoques mediante el uso de redes neuronales artificiales, que agilizan la evaluación en contextos particulares (Da Ponte y Da Silveira, 2008), dado que existen dificultades a la hora de recoger los datos necesarios para analizar el comportamiento de los usuarios frente a las interfaces. Otros enfoques han sido el desarrollo de taxonomías para facilitar la identificación y recogida de las variables asociadas a los datos de interacción de los usuarios con el software, tales como rutas de navegación y agrupación de caracteres, y determinar así el comportamiento de los usuarios durante la interacción con la interfaz (Hanna et al., 2012). Igualmente, la utilización de la Teoría Inventiva para la Resolución de Problemas o TRIZ, que combina reglas del diseño de la interacción, métodos múltiples de evaluación de la usabilidad, recolectores de tendencias de la evolución de la interacción (Filippi y Barattin, 2012), modelos formales de tareas basados en TaMoGolog (Rukh, 2012), la Teoría de la Actividad (Cao et al., 2012), y otros relacionados con la combinación de Teoría del Diseño y los Sistemas de Información en la Interacción Humano-Computador (Spagnoletti y Tarantino, 2013).

Sin embargo, desde el punto de vista de Interfaces para Monitorización y Supervisión Industrial, aun son escasos los aportes sobre metodologías de evaluación de usabilidad, debido a la separación existente entre las operaciones automatizadas de los procesos y su interpretación a través de la interfaz para la toma de decisiones por parte del operador. Una aproximación para tal integración es la metodología de modos de marchas y paros o guía GEMMA (Ponsa et al., 2007) que combina sistemas de eventos discretos DES y actividades humanas, así el operador humano puede cumplir un rol dentro de las acciones de los dispositivos automáticos sobre el proceso. La misma guía es posteriormente aplicada sobre un simulador para el entrenamiento de operadores (Ponsa et al., 2009). Otra propuesta metodológica, involucra nuevas tendencias en supervisión de procesos como aportes preliminares para la construcción de sistemas de supervisión holónicos (ente autónomo y cooperativo) que relacionan aspectos formales (modelos) con aspectos tecnológicos (interfaces de operador) (Zapata et al., 2011), pero no plantea la manera de evaluar la usabilidad de tal conjunción.

Los enfoques anteriores, están orientados a permitir al equipo de desarrollo de software recopilar y analizar automáticamente los usuarios y sus actividades y el comportamiento del sistema para el reconocimiento de problemas de usabilidad y problemas de funcionalidad de interfaces de forma eficiente y eficaz. Como 
consecuencia, se espera que las interfaces se ajusten mejor a la dinámica de los sistemas socio-técnicos en diferentes niveles (es decir, individual, grupal, organizacional, institucional, etc.). Para esto no es posible sin la aplicación de métodos, que permitan soportar la evaluación. Los métodos de evaluación, pueden clasificarse de varias maneras (Granollers, 2004), como se resume en la tabla 1.

En cuanto al lugar de realización, se distinguen dos categorías generales:

a. Los que se realizan en el laboratorio. El objetivo del evaluador, a veces, es comprobar aspectos sin que precise la ayuda de los usuarios. Este tipo de comprobaciones las realiza, normalmente, en su entorno de trabajo o sea en el laboratorio, donde puede además traer usuarios para un estudio particular.

b. Los que se realizan en el entorno natural o habitual del escenario donde se realizan las tareas a evaluar. Esta situación se produce cuando el evaluador realiza su trabajo en el lugar natural donde se realiza la escena, el lugar de interacción habitual del usuario.

En cuanto al tipo de técnica, se distinguen tres categorías:

a. Métodos de inspección: su principal característica es que unos expertos examinan aspectos de la interfaz del sistema relacionados con la usabilidad y la accesibilidad que la misma ofrece a sus usuarios. Estos métodos tienen en cuenta las opiniones, juicios, informes de los inspectores sobre elementos específicos de la interfaz como factor fundamental de la evaluación de la usabilidad. Son populares en el ámbito de las empresas de producción de servicios software, pues permiten identificar, clasificar y contabilizar un gran número de errores potenciales de usabilidad a precio relativamente bajo.

b. Métodos de indagación: La información acerca del agrado del usuario, quejas, necesidades e identificación de requisitos, son informaciones indispensables sobre todo en etapas tempranas del proceso de desarrollo para que proporcionen información relacionada con el uso y las posibilidades de acceso de un producto. Este tipo de métodos se realiza hablando con los usuarios, observándolos, usando el sistema en trabajo real, y obteniendo respuestas a preguntas verbalmente o por escrito.

c. Métodos de test: En estos métodos, los usuarios representativos trabajan en tareas concretas utilizando el sistema (o el prototipo) y los evaluadores utilizan los resultados para ver cómo la interfaz de usuario da soporte a los usuarios con sus tareas.

En cuanto a la automatización, se pueden distinguir entre métodos:

a. Automáticos: son aquellos métodos que disponen de mecanismos (hardware y/o software) que facilitan la comprobación de los aspectos a validar. Los métodos automáticos resultan altamente eficientes puesto que se realizan muy rápidamente y los resultados provienen de los propios parámetros, sin apreciaciones subjetivas. Una de las características que hacen más atractivos a estos métodos es la posibilidad de realizar tests remotamente (Bartek y Cheatham, 2003; Dray y Siegel, 2004) a través de Internet. Su desventaja es que se usan en sistemas ya finalizados y no en etapas tempranas del ciclo de vida del desarrollo.

b. Manuales: al contrario de los métodos anteriores, no disponen de mecanismos que automaticen su realización. Éstos consumen más recursos: tiempo, evaluadores, etc., pero pueden evaluar aspectos que se "salen del patrón" y pueden realizarse en cualquier etapa del desarrollo y con cualquier tipo de prototipo.

Según el tipo de participantes, en la evaluación, se pueden distinguir los siguientes métodos:

a. Con usuarios y/o implicados: Son los métodos en los que se evalúa el sistema con la intervención directa de usuarios representativos, pudiendo también participar en las sesiones personas que sin ser usuarios finales tienen la condición de implicados del sistema.

b. Métodos sin usuarios y/o implicados: Estos métodos los realizan sólo expertos evaluadores con la ayuda de guiones, pautas o documentos que permiten hacer un seguimiento de los que han hecho los usuarios mientras utilizaban el sistema.

Tabla 1: Clasificación de los métodos de evaluación de la usabilidad

\begin{tabular}{|l|l|}
\hline Clasificación de los métodos de evaluación de usabilidad \\
\hline \multirow{2}{*}{ Lugar } & Laboratorio \\
\cline { 2 - 2 } & Entorno real \\
\hline \multirow{3}{*}{ Técnica } & Inspección \\
\cline { 2 - 2 } & Indagación \\
\cline { 2 - 2 } & Test \\
\hline \multirow{2}{*}{ Participantes } & Con usuarios \\
\cline { 2 - 2 } & Sin usuarios \\
\hline \multirow{2}{*}{ Automatización } & Automáticos \\
\cline { 2 - 2 } & Manuales \\
\hline
\end{tabular}

De acuerdo con la anterior clasificación, en este trabajo, la evaluación de la usabilidad de la interfaz de supervisión se realizó en un laboratorio, utilizando métodos de inspección por un experto e indagación a los usuarios y se convalidó con el método automático propuesto como se explica a continuación. 


\section{MATERIALES Y MÉTODOS}

A. Lugar de realización: La metodología se aplicó en dos lugares, una sala de automatización de procesos que funciona como un entorno natural de supervisión de procesos y una sala especialmente equipada para realizar evaluaciones como un laboratorio de usabilidad.

B. participantes: para la evaluación de laboratorio, se utilizaron 12 estudiantes distribuidos en tres grupos y para la evaluación en la sala de automatización, se utilizaron 3 operadores de salas de control de proceso. Los estudiantes, de los programas de ingeniería entre 8 y 10 semestre tienen competencias en procesos industriales relacionados con fluidomecánica, termotransferencia, máquinas, modelamiento, control y simulación de procesos industriales y automatización. Los operadores, por su experiencia trabajaron individualmente. El periodo de experimentación fue de una hora. Además del diseño de la metodología, los autores de este trabajo actuaron como facilitadores de la evaluación.

C. Escenarios: Para la realización de las pruebas, se utilizo un proceso de dosificado de líquidos, donde es posible generar situaciones de manera programada y controlada, las condiciones del proceso cambian gradualmente, a través de la interfaz de supervisión, el operador debe identificar la situación y sus consecuencias antes de tomar las acciones correctivas necesarias.

D. Descripción de la metodología: La metodología de evaluación de Usabilidad de Interfaces de Supervisión desarrollada, consta de un sistema de adquisición de datos para supervisión y control de procesos para el diseño de las interfaces, un sistema de registro de eventos de interacción, un programa clasificador de eventos en criterios de usabilidad y un sistema de inferencia neuro-difuso adaptativo (ver figura 1).

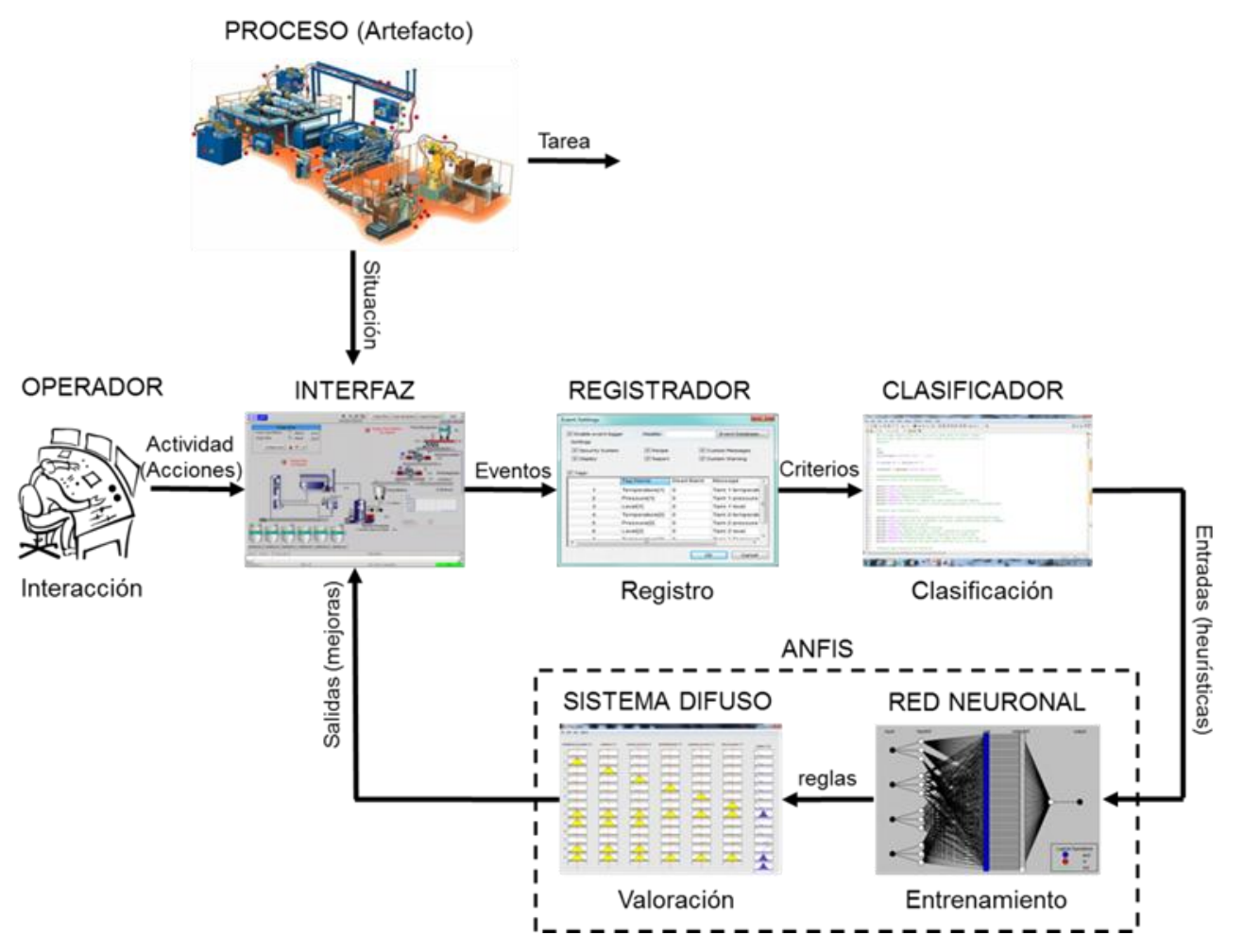

Fig. 1: Esquema general del sistema de evaluación de usabilidad de Interfaces de Supervisión de Procesos

El sistema de adquisición de datos para supervisión y control que se utilizó fue una versión de demostración del paquete InduSoft Web Studio (IWS). IWS, que explota las características fundamentales de los sistemas operativos de Microsoft y permite construir con todas las funciones del sistema de supervisión. Este paquete cuenta con una base de datos de etiquetas de proyecto para gestionar datos en tiempo de ejecución, incluyendo tanto variables internas, como escaneado de E/S para dispositivos sensores y accionamientos; controladores configurables para comunicarse en tiempo real con controladores lógicos programables, dispositivos de E/S remotos y otros dispositivos de adquisición de datos; pantallas de interfaces animadas y cuadros de mando; y módulos opcionales, como registro de alarmas, registro de eventos, registro de tendencias, procedimientos, informes, lógica programable, programadores, un sistema de seguridad, y una completa base de datos de interface. Con este paquete se desarrolló una interfaz de supervisión de un proceso de dosificado de líquidos, con varias pantallas como la que se muestra en la figura 2. 


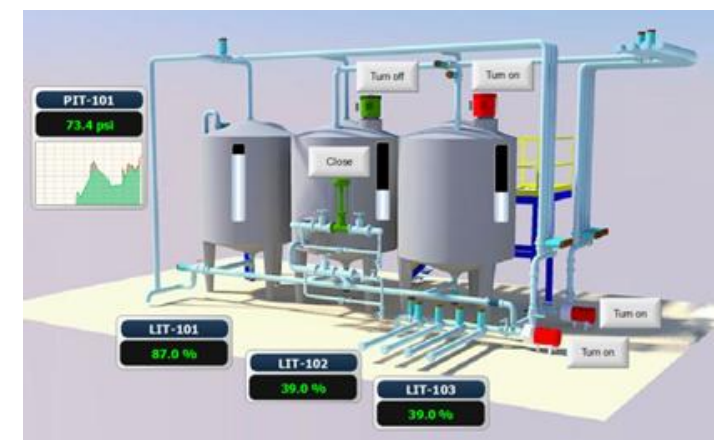

Fig. 2: Pantalla de Interfaz para la supervisión de procesos

El registrador de eventos ofrece la trazabilidad de las acciones del operador durante la interacción con la interfaz o de la actividad interna de la aplicación, como cambios en la seguridad del sistema, operaciones de apertura/cierre de pantallas, operaciones de ejecución de procedimientos o de informes, mensajes personalizados y alertas del sistema. Asimismo, cualquier cambio de valor de etiquetas incluyendo mensajes personalizados (ver figura 3).

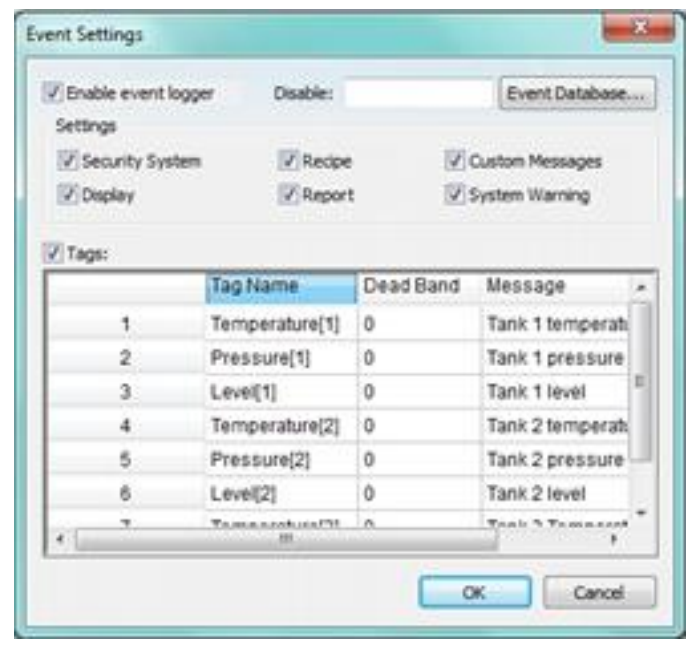

Fig. 3: Módulo de registro de acciones del operador o de actividad interna de la aplicación

Cada acción del operador tiene un código estandarizado como evento, el registro de los eventos es almacenado en archivos de la base de datos del IWS. Un programa explora los eventos correspondientes a las acciones del operador y los clasifica en diez criterios de usabilidad de interfaces definidos en (Seffah et al., 2006) (ver tabla 2). Las acciones tomadas por el registrador de eventos, constituyen una base de conocimiento. Los criterios son utilizados por el sistema de inferencia neuro-difuso adaptativo en forma de vectores. El sistema de inferencia, utiliza la base de conocimiento heurístico de las acciones de los operadores de procesos para el entrenamiento de una red neuronal. El entrenamiento genera un sistema de inferencia difuso, donde cada regla se establece de acuerdo con la distribución normal del número de acciones del operador durante la interacción con el proceso.

Tabla 2: relación de las acciones del operador y los criterios de usabilidad

\begin{tabular}{|l|l|}
\hline \multicolumn{1}{|c|}{ Acciones del operador } & \multicolumn{1}{c|}{ Criterio de usabilidad } \\
\hline Verificación de la comunicación y actualización de datos del proceso & Eficiencia o velocidad de respuesta \\
\hline Seguimiento de una secuencia para el cumplimiento de una tarea & Eficacia \\
\hline Seguimiento de varias secuencias para el cumplimiento de varias tareas & Productividad o multitarea \\
\hline Modificación de pantallas (textos, color y objetos) & Satisfacción o flexibilidad \\
\hline Cantidad de seguimiento de secuencias para el cumplimiento de tareas & Facilidad de aprendizaje \\
\hline Activación de pantallas después de la aparición de una señal de alarma & Seguridad o fiabilidad \\
\hline Tiempo transcurrido sin realizar alguna acción & Confianza \\
\hline Activación de íconos dentro de las pantallas & Accesibilidad o interactividad \\
\hline Modificación de pantallas (textos, color y objetos) & coherencia \\
\hline Reinicio del sistema & recuperación de errores \\
\hline
\end{tabular}


Para cada criterio de usabilidad se han definido tres funciones de pertenencia, baja, media y alta. En este caso se utilizó un sistema difuso que se basa en las reglas de Takagi-Sugeno, que usan una función lineal de las entradas al modelo como consecuente de las reglas. Tal estructura está en capacidad de representar una clase general de sistemas estáticos o dinámicos no lineales. La red neuronal, se entrena con diez capas, cada capa con el número de neuronas correspondientes al número de entradas. La red neuronal utiliza el principio de retropropagación en donde se ajusta el valor de los pesos en funcion del error generado. Esta técnica permite tener un método de optimización que se encuentra al definir el gradiente del error y minimizarlo con respecto a los parámetros de la red neuronal. El sistema completo es utilizado para determinar si la interfaz posee una alta, media o baja usabilidad (como salida del sistema de lógica difusa).

E. Procedimiento: el sistema completo se ejecuta como complemento a las interfaces de supervisión durante la interacción con los operadores durante una hora. El sistema almacena los resultados de la evaluación que luego son comparados con los resultados de la evaluación por los métodos de inspección e indagación.

\section{RESULTADOS Y DISCUSIÓN}

Para el entrenamiento de la red neuronal se utilizaron 100 datos, una capa oculta de 10 neuronas y el algoritmo de retropropagación de Levenberg-Marquardt. Para comprobar la validez de la metodología automatizada (A), se realizó una prueba de hipótesis frente a otras dos metodologías tradicionales: inspección por un experto $(\mathrm{E})$ e indagación a los usuarios $(\mathrm{U})$. Se tabularon los datos de la experimentación de interacción entre los operadores y las interfaces de supervisión de un proceso de control de flujo, nivel, temperatura y presión de líquidos. Las valoraciones arrojadas por el sistema desarrollado, sirvieron para clasificar las interfaces como de baja, media y alta usabilidad. La comparación se muestra en la tabla 3.

Tabla 3: comparación de la valoración de las interfaces según su usabilidad

\begin{tabular}{|c|c|c|c|c|c|c|c|c|c|}
\hline & \multicolumn{3}{|c|}{ Baja usabilidad } & \multicolumn{3}{|c|}{ Media usabilidad } & \multicolumn{3}{|c|}{ Alta usabilidad } \\
\hline O & E & U & A & E & U & A & E & U & A \\
\hline O1 & 8,7 & 8,0 & 8,0 & 9,0 & 8,5 & 7,9 & 8,0 & 8,5 & 8,3 \\
\hline O2 & 8,3 & 8,0 & 9,0 & 8,6 & 8,0 & 8,0 & 8,5 & 8,0 & 8,0 \\
\hline O3 & 8,0 & 8,0 & 9,0 & 8,8 & 9,0 & 9,0 & 9,0 & 8,2 & 8,5 \\
\hline O4 & 8,0 & 8,0 & 9,0 & 8,5 & 8,0 & 9,0 & 8,4 & 8,0 & 8,0 \\
\hline O5 & 8,6 & 8,5 & 9,0 & 8,9 & 8,3 & 9,0 & 8,3 & 8,1 & 8,0 \\
\hline O6 & 8,8 & 8,0 & 9,0 & 8,7 & 8,4 & 9,0 & 9,1 & 8,6 & 8,9 \\
\hline O7 & 7,5 & 7,5 & 7,0 & 8,4 & 8,9 & 9,0 & 8,8 & 8,0 & 8,7 \\
\hline O8 & 8,2 & 7,0 & 7,0 & 8,3 & 8,0 & 9,0 & 9,0 & 8,5 & 8,9 \\
\hline O9 & 8,9 & 7,5 & 7,0 & 9,0 & 8,5 & 9,6 & 9,5 & 9,0 & 9,0 \\
\hline O10 & 8,5 & 7,0 & 7,0 & 8,5 & 8,0 & 9,0 & 9,0 & 9,2 & 9,0 \\
\hline O11 & 8,7 & 8,0 & 8,0 & 9,0 & 9,0 & 8,5 & 8,0 & 9,0 & 8,4 \\
\hline O12 & 8,3 & 8,0 & 9,0 & 8,6 & 9,0 & 8,9 & 8,3 & 9,0 & 8,3 \\
\hline O13 & 8,0 & 8,0 & 9,0 & 8,8 & 9,0 & 8,7 & 8,4 & 9,0 & 9,1 \\
\hline O14 & 9,0 & 8,5 & 8,0 & 9,0 & 8,4 & 8,4 & 8,9 & 9,0 & 8,8 \\
\hline O15 & 9,0 & 8,9 & 8,3 & 9,0 & 9,0 & 8,5 & 8,0 & 9,0 & 8,4 \\
\hline O16 & 9,0 & 8,7 & 8,4 & 9,0 & 9,0 & 8,9 & 8,3 & 9,0 & 8,3 \\
\hline O17 & 7,0 & 8,4 & 8,9 & 9,0 & 9,0 & 8,7 & 8,4 & 9,0 & 9,1 \\
\hline O18 & 9,0 & 8,5 & 8,0 & 9,0 & 7,0 & 8,4 & 8,9 & 9,0 & 8,8 \\
\hline O19 & 9,0 & 8,9 & 8,3 & 9,0 & 8,3 & 8,7 & 8,7 & 8,4 & 9,0 \\
\hline O20 & 9,0 & 8,7 & 8,4 & 9,0 & 9,1 & 8,4 & 8,4 & 8,9 & 9,0 \\
\hline O21 & 7,0 & 8,4 & 8,9 & 9,0 & 8,8 & 8,3 & 8,3 & 8,0 & 9,0 \\
\hline O22 & 8,7 & 8,4 & 9,0 & 8,7 & 8,4 & 9,0 & 9,0 & 8,5 & 9,6 \\
\hline O23 & 8,4 & 8,9 & 9,0 & 8,4 & 7,0 & 8,3 & 8,0 & 9,0 & 9,0 \\
\hline O24 & 8,3 & 8,0 & 9,0 & 8,3 & 7,0 & 9,0 & 8,5 & 9,6 & 9,5 \\
\hline O25 & 9,0 & 8,5 & 9,6 & 9,0 & 7,0 & 8,5 & 8,0 & 9,0 & 9,0 \\
\hline O26 & 7,0 & 8,3 & 8,0 & 9,0 & 7,0 & 8,3 & 8,0 & 9,0 & 9,0 \\
\hline O27 & 7,0 & 9,0 & 8,5 & 9,6 & 7,0 & 9,0 & 8,5 & 9,6 & 9,5 \\
\hline O28 & 7,0 & 8,5 & 8,0 & 9,0 & 7,0 & 8,5 & 8,0 & 9,0 & 9,0 \\
\hline O29 & 9,0 & 8,9 & 8,3 & 8,5 & 8,0 & 9,0 & 9,0 & 8,5 & 8,0 \\
\hline O30 & 8,0 & 9,0 & 9,0 & 8,5 & 8,0 & 9,0 & 9,0 & 8,5 & 8,0 \\
\hline
\end{tabular}

Con los datos obtenidos, se desea suponer que por las valoraciones dadas, los métodos son aproximados. Entonces la hipótesis nula es que el promedio las valoraciones son iguales o estadísticamente existen diferencias significativas que permitan afirmar que el promedio de los tres métodos no es el mismo. Para probar esta hipótesis se elaboraron tablas de Anova. 
En la tabla de Anova, la fuente de variación tiene dos componentes, el factor y el error. Esta fuente de variación se descompone en una suma de cuadrados, la suma de cuadrados del factor, expresada como una diferencia de cuadrados y la suma de cuadrados del error, otra diferencia de cuadrados. Los grados de libertad del factor es $c-1$ y los grados de libertad del error es $n-c$, donde $c$ es el número de columnas (3) y $n$ es el numero de datos (30). Igualmente, se calculó el cuadrado medio como la suma de cuadrados entre el grado de libertad, tanto para el factor como para el error. La última columna se calcula la estadística de prueba $f$, como el cuadrado medio del factor entre el cuadrado medio del error (ver tabla 6 ).

Tabla 6: Operaciones de Anova

\begin{tabular}{|c|c|c|c|c|c|}
\hline Fuente & \multicolumn{2}{|c|}{ Suma de cuadrados } & Grados de libertad & Cuadrado medio & $f$ \\
\hline Factor & & $C_{i}^{2} \quad x^{2}$ & & SCfactor & \\
\hline ractur & sefactor & $\overline{k_{i}}$ & $c-1$ & CMfactor $=$ & CMfactor \\
\hline Error & SCerror $=$ & $x^{2}-\frac{C_{i}^{2}}{k_{i}}$ & $n-c$ & CMerror $=\frac{\text { SCerror }}{n-c}$ & $\overline{\text { CMerror }}$ \\
\hline
\end{tabular}

Haciendo las operaciones y tomando de una tabla de valores críticos de distribución $f$ para un $5 \%$ de área de rechazo, se ubica la relación entre 3 grados de libertad para el factor y 27 grados de libertad para el error, se obtiene un valor crítico de 2,95. La estadística de prueba para la usabilidad baja es 0,09, para media es 2,01 y para alta es 0,38. Esto indica que la hipótesis no se descarta, es decir, en promedio las valoraciones son las mismas. Con ello, se deduce que la metodología propuesta, tiene equivalencia con dos metodologías tradicionales, la inspección de un experto y la indagación a los usuarios, pero tiene la ventaja de que es completamente automatizada. Las tablas de Anova 7, 8 y 9, muestran los cálculos y la estadística de prueba obtenidos para los datos de la evaluación de usabilidad baja, media y alta de las interfaces.

Tabla 7. Anova para usabilidad baja

\begin{tabular}{|c|c|c|c|c|}
\hline Fuente & Suma de cuadrados & Grados de libertad & Cuadrado medio & $f$ \\
\hline Factor & 0,40 & 3 & 0,13 & \multirow{2}{*}{0,09} \\
\hline Error & 38,14 & 27 & 1,41 & \\
\hline
\end{tabular}

Tabla 8. Anova para usabilidad media

\begin{tabular}{|c|c|c|c|c|}
\hline Fuente & Suma de cuadrados & Grados de libertad & Cuadrado medio & $f$ \\
\hline Factor & 6,69 & 3 & 2,23 & \multirow{2}{*}{2,01} \\
\hline Error & 29,95 & 27 & 1,11 & \\
\hline
\end{tabular}

Tabla 9. Anova para usabilidad alta

\begin{tabular}{|c|c|c|c|c|}
\hline Fuente & Suma de cuadrados & Grados de libertad & Cuadrado medio & $\mathrm{f}$ \\
\hline Factor & 0,77 & 3 & 0,26 & \multirow{2}{*}{0,38} \\
\hline Error & 18,32 & 27 & 0,68 & \\
\hline
\end{tabular}

Es importante aclarar, que esta metodología está en función de la interacción con el operador, pero el comportamiento del operador puede obedecer a otras variables no tenidas en cuenta en la metodología. Por ejemplo, los operadores pueden manifestar diversos comportamientos o realizar varias actividades que no están ligadas directamente con el proceso, incluso, la actividad de interacción con las pantallas de una interfaz puede generar aburrimiento entre los operadores. Estos elementos aunque importantes no son considerados dentro de esta metodología.

El desempeño del operador durante la interacción a través de la interfaz, tiene una relación directa con el conocimiento que tenga del proceso, haciéndolo más eficaz o menos productivo a la hora de realizar una tarea y/o tomar decisiones. Varias de las tareas asignadas a los operadores se rigen bajo ciertos patrones de secuencias a seguir, luego, depende del operador y su habilidad para memorizar y realizar $n$ veces la misma acción, la cantidad de veces que se siga la misma secuencia en determinado tiempo. Los trabajos futuros están orientados a utilizar la metodología aquí presentada en varias aplicaciones, incluso de otros campos diferentes a la supervisión de procesos industriales, dado que actualmente existe un mercado diverso de productos con un alto grado de interacción con el usuario. Igualmente, para que el proceso evaluación de usabilidad de interfaces y/o productos interactivos, es importante la incorporación de buenas 
aplicaciones informáticas para la captura y análisis de registro de datos, por ello, este también es un campo de exploración.

\section{CONCLUSIONES}

De acuerdo con los resultados obtenidos se pueden extraer las siguientes conclusiones:

La usabilidad es reconocida como un factor de calidad importante para sistemas interactivos incluidas las interfaces de usuario de una gran variedad de dispositivos. El diseño de aplicaciones, de modo que logren efectivamente sus propósitos en términos de facilidad de uso, no es una tarea fácil, ante la existencia de varios métodos y técnicas para la evaluación de la usabilidad que no ha sido posible integrarlas en un sencillo marco conceptual que facilite su uso por los desarrolladores. La metodología desarrollada, hace parte de las técnicas automatizadas de acuerdo con la clasificación presentada en este artículo. Sin embargo, incorpora a los usuarios (operadores) para extraer información durante la interacción en tiempo de ejecución en actividades de supervisión de procesos, lo cual constituye una innovación frente a técnicas tradicionales del mismo tipo.

La literatura tiende a definir la usabilidad en términos excesivamente breves y ambiguos y describen su aplicación en términos informales. Esta es una de las razones principales por las cuales predominan los diferentes enfoques y orientaciones en el estudio de la usabilidad. Aunque no existe una concepción universal sobre criterios reales de evaluación de la usabilidad de sistemas interactivos, con la aproximación presentada por varios autores, se logró establecer una relación entre las acciones del operador sobre la interfaz y los eventos de interacción establecidos en el software de supervisión de procesos.

Con la estadística de prueba, se determinó que la metodología es eficiente para actividades de interacción con interfaces de usuario, porque utiliza elementos que se encuentran incorporados y probados en el software de supervisión y se complementan con una técnica de inteligencia artificial que igualmente ha mostrado sus bondades en otras aplicaciones.

El objeto de la evaluación de usabilidad de interfaces es utilizar los resultados para realizar una retroalimentación, con el fin de mejorar los diseños y por ende la interactividad con el usuario. La metodología desarrollada le permite a un inexperto en usabilidad agilizar la evaluación para proponer las mejoras que den lugar a la optimización de la interacción con los procesos.

\section{AGRADECIMIENTOS}

Los resultados hacen parte del proyecto "Desarrollo de una metodología de evaluación de usabilidad de Interfaces Humano-Máquina (IHM) para la mejora del proceso de toma de decisiones en tareas de supervisión industrial", financiado por la Universidad Nacional de Colombia, Sede Manizales.

\section{REFERENCIAS}

Anderson, J. R. Learning and Memory, 2ª edición, Wiley,Nueva York, Estados Unidos (2000).

Ankita, M. y Sanjay, K. Usability Evaluation Methods: A Literature Review. International Journal of Engineering Science and Technology, 4(2), (2012).

Bartek, V. y Cheatham, D. Experience remote usability testing, part 1: Examine study results on the benefits and downside of remote usability testing (2003). En URL: http://www.ibm.com/developerworks/library/warmusts1 (2012).

Cao, X., Lijue, W. y Shijian, L. Research on Contextual Design in Human-Computer Interaction Under the Framework of Activity Theory. Proceedings of the 2012 International Conference on Information Technology and Software Engineering. Lectures Notes in Electrical Engineering, 11, 853-868 (2012).

Da Ponte, M. y Da Silveira, A. A Methodology for Evaluation the Usability of Software for Industrial Automation Using Artificial Neural Networks: Case Study-Eletrobrás. 2008 International Conference on Computational Intelligence for Modeling, Control and Automation. 430-435 (2008).

Dix, A., Finlay, J., Abowd, G. y Beale R. Human-Computer Interaction. Pearson Education Ltd. 3ª edición (2004). 
Dray, S. y Siegel, D. Remote possibilities?: international usability testing at a distance. Interactions, 11(2), 10-17, (2004).

Filippi, S y Barattin, D. Integrating Systematic Innovation, Interaction Design, Usability Evaluation and Trends of Evolution. Sustainable product development, CIRP Design 2012. Editor Amaresh Chakrabarti. p. 301-311 (2012).

Granollers, T. MPlu+a. Una metodología que integra la ingeniería del software, la interacción persona ordenador y la accesibilidad en el contexto de equipos de desarrollo multidisciplinares. Tesis Doctoral. Departament de Llenguatges i Sistemes Informàtics, Universitat de Lleida, España (2004).

Hanna, N., Richards, D. y Jacobson, M. Automatic Acquisition of User Models of Interaction to Evaluate the Usability of Virtual Environments. Knowledge Management and Acquisition for Intelligent Systems. Lectures Notes in Computer Science. 7457, 43-57 (2012).

ISO 9241. Ergonomic requirements for office work with visual display terminals (1998).

ISO 9241-11, Requisitos ergonómicos para trabajos de oficina con pantallas de visualización de datos (PVD), Parte 11: Guía sobre usabilidad, $1^{\text {a }}$ edición, Navarra, España (1998).

ISO/IEC 25000:2005. Software Engineering - Software product Quality Requirements and Evaluation (SQuaRE), (2005).

Lorés, J. Julio Abascal, J., Aedo, I., Cañas, J., Gea, M., Belén, A. G., Belén, A. M., Ortega, M., Valero, P. y Vélez M. Introducción a la Interacción Persona-Ordenador. $1^{\underline{a}}$ edición, Editor Jesús Lorés, (2001).

Granollers, T., Lorés, V., Cañas, J. Diseño de sistemas interactivos centrados en el usuario. Editorial UOC, Colección Informática, no 43, p. 168 (2005).

Norman, D. Cognitive engineering. $1^{\text {a }}$ edición, 32-65, D. A. Norman \& S. W. Draper editors, Nueva Jersey, Estados Unidos (1986).

Ponsa, P., Vilanova, R. y Díaz, M. Introducción del Operario Humano en el Ciclo de Automatización de Procesos Mediante la Guía GEMMA. Información Tecnológica, 18(5), 21-38 (2007).

Ponsa, P., Amante, B. y Díaz, M. Evaluación de la usabilidad para la tarea de supervisión humana en sala de control industrial. Revista RIAI Revista Iberoamericana de Automática e Informática Industrial, 6(1), 8493, (2009).

Rodeiro, J., Representación y Análisis de la componente visual de la interfaz de usuario, Tesis doctoral. Universidad de Vigo, España (2001).

Seffah, A., Donayaee, M., Kline, R., Padda, H. Usability measurement and metrics: A consolidated model, Software Quality Control, 14(2), (2006).

Rios, D., Vasquez, A., Mosqueira, E. y Moret, V. Usability: A Critical Analysis and a Taxonomy. International Journal of Computer-Interaction 26(1):53-74 (2010).

Rukh, H. Incorporating Usability Evaluation in Software Development Environments. KI-künstliche Intellinez. 26(2), 197-200 (2012).

Spagnoletti, P. y Tarantino, L. User Centered Systems Design: The Bridging Role of Justificatory Knowledge. Designing Organizational Systems. Lectures Notes in Information Systems and Organization, 1, 105-121 (2013).

Wickens, C. y Hollands, J., Engineering Psychology and Human-Computer Performance, $3^{\text {a }}$ edición, 513550, Prentice Hall, Nueva Jersey, Estados Unidos (2000).

Zapata, G., Cardillo, J. y Chacón, E. Aportes Metodológicos para el Diseño de Sistemas de Supervisión de Procesos Continuos. Información Tecnológica, 22(3), 97-114 (2011). 
